\title{
MULTIPARAMETER SPECTRAL THEORY
}

\author{
BY F. V. ATKINSON
}

1. Introduction. On an occasion calling for a topic of general mathematical interest, I owe my audience some explanation for having chosen one of the less known areas of analysis as my subject today. The idea of considering eigenvalue problems with several parameters occurs quite naturally in certain boundary-value problems, in particular those which lead to the Mathieu functions; the area in question is thus not unknown to the applied mathematician or the specialist in the "special functions of analysis." However, the exploitation of this area in the spirit of classical analysis, in particular for ordinary differential equations, has reached no more than a preliminary stage. The same is true when we examine this area in the spirit of linear algebra or again of functional analysis. It is when we look at this area in a more abstract way that another justification appears for recommending it as worthy of general interest. The formal theory of eigenvalue problems with several parameters makes extensive use of algebraic ideas which, though not new, have gained great vogue in recent years.

The spectral theory of linear operators has tended to be concentrated to an overwhelming degree on the study of endomorphisms, vis-à-vis the identity. In what I shall refer to as the standard case, one has a linear space $G$, usually over the complex field, and a linear operator $A$ on $G$ into itself. In the spectrum, we study the nature of the linear combination $A-\lambda I$, where $I$ is the identity, and $\lambda$ a complex scalar. This has, of course, been the subject of an enormous literature and has applications too numerous to mention.

Here I am concerned with two generalisations, neither particularly new, but only now beginning to claim their due share of attention. In one of these we allow nonlinear dependence on the scalar parameter, in particular, polynomial or rational dependence. In the matrix context, this is the topic of $\lambda$-matrices (see e.g. [67]). For the differential equations context, there is early work of R. E. Langer [64], [65] and a good deal of more recent work [1], [35], [58], [60], [63], [73]. In the operator context, work of a more spectral character includes [60], [63], [68], [73].

The second generalisation is that in which we introduce several

An address delivered before the Iowa City meeting of the Society on November 27, 1965, by invitation of the Committee to Select Hour Speakers for Western Sectional Meetings; received by the editors August 14, 1967. 
parameters, and several operator functions of them, rather than a single linear function. It is this that properly deserves the term "multiparameter." Work in this area has naturally concentrated on the case of linear parameter dependence and will be briefly surveyed in §3. It is appropriate to take these two generalisations together. For one thing, the two generalisations of the standard case can be combined, as when we consider several operators dependent polynomially on several parameters. For another, they both raise difficulties in connection with the algebraic manipulation of operators, in particular, in connection with the formulation of the functional calculus.

2. The linear multiparameter case. I first recall a preliminary generalisation, in which the standard case appears as a specialisation from the theory of "pencils." In the latter, one has a pair of linear spaces $G, H$, both over the same field $K$, and a pair of linear operators $A, B: G \rightarrow H$. Here spectral theory is concerned with the behaviour of $A \lambda+B \mu$, for varying scalar $\lambda, \mu$, not both zero; however even in the matrix case, other concepts are also relevant. Here the standard case is recovered if we choose $G=H, B=-I$. More generally, we have essentially the standard case if some linear combination of $A, B$ is an isomorphism, which of course need not always be so. For the finitedimensional case one may cite [40], [42], [61], [88], and more generally the recent works [3], [4], [39], [57].

As a generalisation of the topic of pencils, and so also of the standard case, we propound the multiparameter linear eigenvalue problem. For some $k \geqq 1$, we suppose given $k$ pairs of linear spaces $G_{r}, H_{r}$, $r=1, \cdots, k$. Also given is an array of $k(k+1)$ linear operators

$$
\begin{array}{ccc}
A_{10} & \cdots & A_{1 k} \\
\cdots & \cdots & \cdots \\
A_{k 0} & \cdots & A_{k k},
\end{array}
$$

where the operators in any one row have the same action, namely,

$$
A_{r s}: G_{r} \rightarrow H_{r}, \quad r=1, \cdots, k, \quad s=0, \cdots, k .
$$

Of course, we get the case of a pencil if $k=1$.

The pure mathematician, in the absence of prior motivation, will naturally ask whether any problems can be posed for this situation. In particular, since the operators in different rows of the array (2.1) have quite separate actions, one wonders whether they can sensibly be made the ingredients of any unified problem at all. There are at least two answers to this. 
In the first, more superficial problem, no additional machinery is needed. In what may be called the linked eigenvalue problem, we set up the $k$ linear combinations

$$
\sum_{s=0}^{k} A_{r s} \lambda_{s}, \quad r=1, \cdots, k .
$$

Here the $\lambda_{\boldsymbol{s}}$ are scalars, not all zero; it is often convenient to adopt a homogeneous or projective notation for the scalar parameters. It is then natural to define an eigenvalue as a $(k+1)$-tuple $\lambda_{0}, \cdots, \lambda_{k}$ of scalars, not all zero, such that all $k$ of the linear operators (2.3) are singular in the sense of having nonzero kernels. Addicts of index theory would ask also whether these operators had nonzero cokernels, and so on, but let us pass over these ramifications. One emerges with a preliminary notion of the spectrum of the array (2.1) as a collection of (projectively considered) scalar $(k+1)$-tuples.

However, we obviously cannot stop short at a definition of eigenvalues and ancillary notions. One will ask whet the eigenvectors are and whether they have any completeness properties. Here we are constrained to the first of many algebraic constructions. We must consider the tensor product

$$
G=G_{1} \otimes \cdots \otimes G_{k} .
$$

On this space, the operators (2.1) will induce maps into $k$ other spaces, namely,

$$
H_{1} \otimes G_{2} \otimes \cdots \otimes G_{k}, \cdots, G_{1} \otimes \cdots \otimes G_{k-1} \otimes H_{k} .
$$

At the moment we leave open the question of whether we are using the algebraic tensor product or some completion thereof but assume that the tensor product has certain natural properties (validity of the Künneth tensor formula) [46], [48], [66], [72].

Let now $A_{r s}^{\dagger}$ denote the operator induced by $A_{r s}$, acting from (2.4) to the $r$ th space in (2.5). We may formally extend our idea of an eigenvalue by saying that it is a nonzero $(k+1)$-tuple $\lambda_{0}, \cdots, \lambda_{k}$ such that the $k$ operators

$$
\sum_{s=0}^{k} A_{r s}^{\dagger} \lambda_{s}, \quad r=1, \cdots . k,
$$

have kernels with a common, nonzero intersection; there must be a $g \in G, g \neq 0$, annihilated by all of (2.6). This will be generated by tensor products of the separate kernels of (2.3), though here we touch on more delicate matters. We claim that elements of the common ker- 
nel of (2.6) provide a natural notion of an eigenvector and that completeness properties of eigenvectors are to be sought in the tensor product space (2.4), if at all.

It is sometimes asked whether we cannot combine the separate operator-expressions within the simpler context of a direct sum (as for example in [2], [34]) rather than in the more abstruse tensor product. A crude indication that the latter is appropriate to the problems under consideration is given by finite-dimensional situations.

Let in fact

$$
\operatorname{dim} G_{r}=\operatorname{dim} H_{r}<\infty, \quad r=1, \cdots, k,
$$

and let the field be algebraically closed and of characteristic zero. By choosing bases in $G_{r}, H_{r}, r=1, \ldots, k$, we may attach a sense, as polynomials, to the determinants

$$
\operatorname{det} \sum_{s=0}^{k} A_{r s} \lambda_{s}, \quad r=1, \cdots, k .
$$

An eigenvalue, whether for (2.3) or (2.6), will be a common nontrivial zero of these polynomials. In general [89], there will be $\Pi \operatorname{dim} G_{r}$ of these, so that we should get this number of eigenvectors. One then notices that this happens to be the dimension of the tensor product (2.4), so that we have at least the correct number of eigenvectors, apart from degenerate cases. Against this, the dimension of the direct sum is the sum $\sum \operatorname{dim} G_{r}$.

In the ultra-trivial one-dimensional case, an eigenvalue is a simultaneous zero of the linear functions (2.3). In general, there will be essentially one zero, and the eigenvector is just a basis vector in the one-dimensional tensor product.

The general finite-dimensional case, under assumptions of a hermitian character, was considered in [9]. I refer later to work of Carmichael on this topic.

3. Some history. In the standard case it is surely right to view eigenvalue problems for differential equations as second only to eigenvalue problems for matrices in general importance and of primary importance in the inspiration of deeper developments. In the multiparameter case, the matrix and differential equation problems themselves stand in need of much development but will no doubt in time provide useful inspiration for more abstract settings of such problems.

However, in the differential equation setting multiparameter problems do have an antiquity not far short of Sturm-Liouville theory 
itself. Let us formulate the multiparameter analogue of the classical Sturm-Liouville problem. We seek $k$ functions $y_{r}\left(x_{r}\right), a_{r} \leqq x_{r} \leqq b_{r}$, satisfying $k$ second-order differential equations

$$
y_{r}^{\prime \prime}\left(x_{r}\right)+\left(\sum_{s=1}^{k} \lambda_{s} p_{r s}\left(x_{r}\right)+q_{r}\left(x_{r}\right)\right) y_{r}\left(x_{r}\right)=0 ;
$$

here we have reverted to inhomogeneous spectral parameters. For the boundary problem, we are to determine the $\lambda_{1}, \cdots, \lambda_{k}$ such that all equations (3.1) have nontrivial solutions satisfying boundary conditions of the usual type; for simplicity, we suppose these to be

$$
y_{r}\left(a_{r}\right)=y_{r}\left(b_{r}\right)=0, \quad r=1, \cdots, k .
$$

This problem is discussed in the book of Ince [55] (see also [16][18], [25], [50], [51], [59], [79], [93]-[94]). In addition to the $p_{r s}, q_{r}$ being real-valued and well-behaved, let there hold (as in the standard case) the definiteness assumption

$$
\operatorname{det}\left\{p_{r s}\left(x_{r}\right)\right\}_{r, s=1}^{k}>0, \quad a_{r} \leqq x_{r} \leqq b_{r}, \quad r=1, \cdots, k .
$$

Then all eigenvalues (inhomogeneous $k$-tuples) are real. As in Sturm's oscillation theorem, one has now "Klein's oscillation theorem" according to which an eigenvalue can be chosen uniquely so as to make the $y_{r}\left(x_{r}\right)$ have assigned numbers of zeros in $a_{r}<x_{r}<b_{r}$.

So far we have discussed the "linked eigenvalue problem" for (3.1-3.2). In order to get eigenfunctions with possible completeness properties we must form products $\prod y_{r}\left(x_{r}\right)$ of eigenfunctions of the separate problems. In this way each eigenvalue yields a function of $x_{1}, \cdots, x_{k}$. This is, of course, a tensor product construction. We then consider the completeness of these products in suitable spaces of functions of the $x_{r}$ in the box $a_{r} \leqq x_{r} \leqq b_{r}, r=1, \cdots, k$. The case $k=2$ was discussed by Hilbert (5.1), who is also largely responsible for the algebraic machinery to be introduced later. The general case has been considered by Faierman [37].

The situation (3.1), with boundary conditions of the type (3.2) or other types, arises in the separation of variables technique for partial differential equations and so by a reversal of the tensor product construction. In the simplest case, such as that of the oscillations of a rectangular membrane with fixed boundary, we are led to two separate Sturm-Liouville problems, which are separate not only as regards their independent variables but also in regard to the spectral parameters as well; we have a sort of diagonal situation. In the next most simple case, exemplified by the case of a circular membrane, we 
have mild parametric coupling or a triangular situation. The parameter in the angular equation must be adjusted for periodicity and the resulting values substituted in the radial equation leading to various Bessel functions. We get the multiparameter situation in full force in the case of an elliptic membrane; here the separated equations both contain the same two parameters. It is natural that the theory of Mathieu, Lamé and allied functions has provided a continuing thread, if not the only one, in the topic of multiparameter problems [6], [7], [36], [69], [84], [91].

There are many variations on (3.1-3.2). Two-point boundary conditions can be used; periodic conditions are important for the Mathieu functions. It would be of interest to consider cases in which some or all of the intervals $\left(a_{r}, b_{r}\right)$ are infinite or otherwise "singular." If however these intervals are all finite, we may locate them on the same $x$-axis and consider that we are dealing with a single differential equation containing $k$ parameters and defined on $k$ nonoverlapping intervals on the $x$-axis. Ince [55] does this. An interesting case is that of a single differential equation of the second order containing $k$ parameters, defined in a single interval, for which we demand that there exist a nontrivial solution which vanishes at $k+1$ assigned points of the interval. The case $k=1$ is the usual one. The case $k=2$ is a nontrivial extension considered by Arscott [5].

In a modification of Sturm-Liouville theory of importance for the special functions such as the Legendre polynomials, we have an algebraic differential equation with singularities; instead of boundary conditions of the standard type, we ask that the solution behave in polynomial or related fashion at the singularities. Such problems can be posed with several parameters and singularities [36], [59], [90], [91].

In a related area is the finite-difference analogue of Sturm-Liouville theory, which is manifested in the three-term recurrence relation for orthogonal polynomials. For the multiparameter extension and its connection with orthogonal polynomials in several variables, I refer to [8], [11]. There is an application of completeness in this discrete case to the proof of completeness in the continuous case [37]. The completeness in the discrete case, when considered over a finite range, may be considered as a special case of the matrix problem [9].

Leaving now the Sturm-Liouville area, one may remark that just about any spectral problem involving a single parameter will have nontrivial extensions involving several parameters and a corresponding number of eigenvalue problems. Indeed, we can couple together, by means of spectral parameters, eigenvalue problems of quite differ- 
ent kinds. It was indicated by R. D. Carmichael [23]- [25] that there is here a vast area awaiting investigation; Carmichael also recognised the matrix problem and suggested a possible line of attack on it.

For specific work of this kind, one may mention that of A. J. Pell [78] on pairs of Fredholm integral equations, coupled by a pair of parameters. First-order partial differential equations were considered in $[21],[22],[32]$.

As already mentioned, multi-parameter problems in the full sense arise when we separate the variables in a partial differential equation, without at the same time managing to separate the spectral parameters. Even when we do manage to separate the parameters as well as the variables, there is still something left to discuss. Namely, we have the problem of whether the eigenfunctions obtained by separating the variables form a complete set. In abstract terms, we are concerned with whether the spectral resolution of the Kronecker sum (or product) of a pair of operators is given in the natural way in terms of their separate spectral resolutions (for the matrix case see [13]). For related work see $[14],[15],[20],[26],[29],[30],[41]$. We are in this case concerned with partially diagonal cases of (2.1).

In yet another ramification, not to be considered here, we are concerned with skew-symmetric products, determinants in Banach spaces, and the like. These arise from special symmetric forms of (2.1) in which all the spaces are the same [56], [82], [85].

4. Algebraic aspects. The topic of eigenvalues, eigenfunctions and their completeness, as applied to the immense variety of special multiparameter eigenvalue problems, provides an endless field for investigation. To give only one quite natural example, the detailed behaviour of eigenvalues and eigenfunctions in the multiparameter Sturm-Liouville case is still far from clear. Tempting as is this area for a classical analyst, I would nevertheless like to speak of other matters today, namely the rounding out of the algebraic formalism.

I recall that in the standard case of an endomorphism $A$, an important part is played by a rather simple device, namely the formation of polynomials in $A$ with scalar coefficients; more generally, we can also form rational functions of $A$. Three areas of application of this device may be noted.

In the first, one notes that the polynomials in $A$ form a commutative algebra of endomorphisms. Under normed conditions this leads to the construction of Banach algebras [74], [80], and, under more special conditions, to symmetric algebras and proofs of spectral resolutions [74]. Again, polynomials in the endomorphism can be 
used to give quite elementary proofs of spectral resolutions in Hilbert space. Yet another application of polynomials is to construct by limiting processes a natural interpretation for more general functions of an endomorphism, as in spectral mapping theorems.

The second application of polynomials in an endomorphism is even more basic, though it may be disregarded in many important cases. I refer here to the ideas of root-spaces, adjoined or principal vectors or of the rank of an eigenvalue. Even in the matrix case we must consider not only the eigensubspace, that is to say the kernel of $A-\lambda I$ for an eigenvalue $\lambda$, but also the kernels of $(A-\lambda I)^{n}, n \geqq 1$, whose union forms the root-space; generally, these play an essential role in expansions associated with nonselfadjoint operators [1], [35], [44], [58], [73]. These root-spaces and associated quantities present interest also in themselves, even when we have little hope of establishing completeness properties, and merely wish to clarify the pointwise and local behaviour of $A-\lambda I$ and its inverse [43], [57], [86], [87]. The relevant point at the moment is that this idea of a root-space does not have an immediately obvious extension to the array (2.1), or even to the pencil

$$
A \lambda+B \mu
$$

The "operator-bundle"

$$
\sum_{r=0}^{n} A_{r} \lambda^{n-r} \mu^{r}
$$

presents similar difficulties.

I have mentioned that in certain cases one may reduce (4.1) to the standard case. The same is true of (4.2) which, again under certain restrictions, may be replaced by a problem linear in the paramters, but posed in a direct sum space, namely the direct sum of $n$ copies of the original one [44], [70] much as an $n$th order ordinary differential equation is reduced to a 1st order system. However, even when this reduction to the standard case is possible, it is perhaps also useful to have a formalism which avoids preliminary transformations.

I come now to the third field of application of polynomials, in which it may not be apparent that polynomials are actually involved. I refer here to the topic of products, orthogonality and biorthogonality. For an endomorphism $A$ there holds a biorthogonality between eigenvectors in the given space and those in the dual or conjugate space when these are associated with distinct eigenvalues. In Hilbert space, for a symmetric endomorphism, this leads to an orthogonality between eigenvectors in the given space associated with distinct eigen- 
values. It should now be indicated that this same orthogonality or biorthogonality, as the case may be, also holds when we insert in the product the endomorphism $A$, or for that matter any polynomial in $A$. In this standard case, we naturally tend to emphasise the special inner or scalar product associated with the identity endomorphism; this is particularly so in Hilbert space, where this scalar product is used to define the topology and to select the class of symmetric operators or principal interest.

For (4.1), to take only the simplest case, we do not have an identity operator or a distinguished product. Nevertheless, there holds a rather obvious biorthogonality. If $A, B: G \rightarrow H$, then both of $A, B$, as also any linear combination, gives a product between elements of $G$ and of $H^{*}$. Furthermore, if $\left(A \lambda_{1}+B \mu_{1}\right) g=0, h^{*}\left(A \lambda_{2}+B \mu_{2}\right)=0$, $\lambda_{1} \mu_{2} \neq \lambda_{2} \mu_{1}$, then $h^{*} A g=h^{*} B g=0$, so that we have a whole family of biorthogonalities.

In specialising the latter in the hermitian direction, we do not need to assume that $A, B$ are endomorphisms of a Hilbert space. Let us assume rather that, with the complex field, we have conjugate linear maps $\phi: G \rightarrow H^{*}, \psi: H \rightarrow G^{*}$, such that, for $g \in G, h \in H, \phi(g) h=(\psi(h) g)^{-}$. By saying that $A$ is formally symmetric we are now asserting commutativity in the diagram

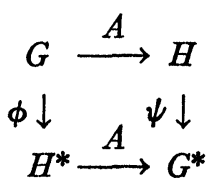

where $A$ has been used to denote the induced map $H^{*} \rightarrow G^{*}$. Then $A$ defines a real-valued quadratic form $\phi(g) A g=\psi(A g) g$ on $G$ as will $B$ if the analogue of (4.3) holds. There is no place here for a uniquely favoured quadratic form or metric, definite or otherwise.

The principal step in setting up the corresponding machinery for such cases as (2.1), (4.1-4.2) lies in studying maps induced by the expressions concerned on modules over polynomial rings. Thus, to take (2.1), we should not immediately pass to the expressions (2.3) or (2.6), considered as operator-valued functions of scalar variables. Instead, we replace the scalars $\lambda_{s}$ by indeterminates to be denoted by $\xi_{8}$. The resulting expressions can still be considered as operators, acting now not on the original spaces, but rather on polynomials in the $\xi_{\text {s }}$ with suitable vectorial coefficients. In all this we are dealing with modules over the ring, or algebra $P$ of polynomials in $\xi_{0}, \cdots, \xi_{k}$ with scalar coefficients. 
We can take a small step back from algebra and in the direction of analysis by going over to a dual machinery which uses the language of partial differential equations. The next few sections will use such a presentation. However one must ultimately address oneself also to the more purely algebraic formalism.

To indicate in a few words the relevance of this machinery to the three areas of application of polynomials listed above, I start with the observation that we are concerned with a collection of solutions of partial differential equations, associated in a rather obvious way with the given operator expressions. We then study the action on this collection effected by linear constant scalar coefficient partial differential operators. Such operators form in various ways an algebra of endomorphisms, and give a functional calculus. One obtains a more flexible approach to eigenvalues by asking what such operators annihilate some of the solutions in this collection; eigenvalues are thus naturally associated with maximal graded ideals in the polynomial algebra $P$ and root-spaces with powers of such ideals. Furthermore, other kinds of ideals also rank for consideration, namely prime, primary, and principal ideals.

As to products, orthogonality, and so on, a rather complicated but none the less interesting situation emerges. So far as the general theory is concerned, and apart from the ad hoc construction of products in particular cases, we must start with the observation that we can form the tensor product $P \otimes P$ of $P$ with itself over the field; effectively, we are forming the polynomial algebra in double the number of indeterminates. The second factor $P$ might be considered as an opposite algebra, though this point is not material in the commutative case. We are then concerned with the fact that $P$ can be considered as a $P \otimes P$-module and the resulting relations between tensor products and torsion modules for supplemented algebras [27].

5. Partial differential equations. A common line of argument is to deduce results concerning the solutions of constant coefficient, partial differential equations from information about the behaviour of associated operator-valued functions derived by replacing the partial differentiations by scalar variables; the connection between the two in this direction may be established, for example, by the Fourier transform. In particular, the spectral resolution of an endomorphism gives rise to explicit solutions of associated differential equations. Here our point of view is different. We are interested in using partial differential equations as a language covering algebraic manipulations for arrays such as (2.1). The use of differential processes for the discussion of operator-bundles such as (4.2) may be consulted in [60]. 
Let us suppose that we have $k$ expressions (the number is not actually crucial at the moment)

$$
D_{r}\left(\xi_{0}, \cdots, \xi_{k}\right), \quad r=1, \cdots, k,
$$

which are polynomials in the indeterminates $\xi_{s}$, with coefficients being linear operators from a linear space $G_{r}$ to a linear space $H_{r}$, respectively. For definiteness, we will assume that these polynomials are homogeneous. The next step is to replace them by similar expressions

$$
D_{r}^{\dagger}\left(\xi_{0}, \cdots, \xi_{k}\right), \quad r=1, \cdots, k,
$$

where the operator-coefficients in $D_{r}$ have now been given their induced actions on the tensor product $G$ as in $\$ 2$. We then form the linear constant coefficient partial differential operators

$$
D_{r}^{\dagger}\left(\partial / \partial x_{0}, \cdots, \partial / \partial x_{k}\right), \quad r=1, \cdots, k .
$$

If the partial differentiations are interpreted in the usual way, we may consider the action of (5.3) on any linear space of infinitely differentiable functions with values in $G$. However, a similar apparatus may be used quite generally, in the absence of any topology in the field. We consider the set $Q(G)$, say, of polynomials or formal power series in the $x_{*}$ with coefficients in $G$, and let the partial differentiations act formally. We then define the set $N$ of solutions of the simultaneous equations

$$
D_{r}^{\dagger}\left(\partial / \partial x_{0}, \cdots, \partial / \delta x_{k}\right) u=0, \quad r=1, \cdots, k, \quad u \in Q(G) .
$$

We may grade $N$ into homogeneous components $N^{(t)}$, say, being the set of solutions of (5.4) which are homogeneous polynomials of degree $t$ with coefficients in $G$.

The objects $Q(G), N$ are $P$-modules in the sense that $P$ is the algebra of scalar constant coefficient, partial differential operators. It is an essential observation that $N$ is closed under partial differentiations.

It is of interest to study, in the finite-dimensional case, the dimensionality of the space formed by the set of homogeneous $n$th degree elements of $N$. This integer-valued function of $n$ forms a sort of Hilbert function [47]. We are mainly concerned with the case in which, for large $n$, this function is constant. For work on polynomial solutions of constant coefficient equations, I cite [38], [54], [75].

In the infinite-dimensional case we can no longer talk about dimension. Instead, so far as constant dimension is concerned, we ask 
whether there is an isomorphism between graded components of $N$ of different degrees, effected by a homogeneous element of $P$, perhaps of the first order. More generally, we consider reduction by normal sequences [76].

In the case of (4.2) we get an $n$th order differential operator, which annihilates trivially polynomials of degree less than $n$. In this case it is of interest to consider polynomials in $N$ of degree $n-1$ and up, with particular emphasis on the lowest component. In the standard case this amounts to emphasising the original domain space.

6. The functional calculus. We express this in terms of the set $N$ of polynomial solutions of (5.4) with vector coefficients. If $p \in P$ is a homogeneous scalar partial differential operator, then $p$ maps $N$ into itself; it is an endomorphism of $N$ as a graded $P$-module, lowering the degree of graded components by the order of $p$ as a differential operator. With certain reservations, we can attach a sense also to the inverse operator $p^{-1}$ and to quotients $p_{1} / p_{2}$ of homogeneous elements of $P$.

To be more specific, let us say that $p \in P$, of order $m$ say, is "regular" if the map of graded components

$$
p: N^{(t+m)} \rightarrow N^{(t)}
$$

is an isomorphism (of linear spaces) for $t \geqq t_{0}$ and some integer $t_{0}$. The set of regular elements of $P$ includes the nonzero scalars and is closed under multiplication and factorisation. One can thus say that an element is regular if and only if it does not lie in a certain set of (homogeneous) prime ideals in $P$; this gives us a natural notion of the spectrum.

For the functional calculus we consider "admissible" fractions $p_{1} / p_{2}$; here $p_{1}, p_{2}$ are to be homogeneous elements of $P$ of the same degree, with $p_{2}$ "regular" in the above sense. With the usual operation on formal fractions or quotients and rules for mutual identification, we can say that the collection of admissible fractions forms an algebra over the field in question. We denote this algebra by $R$ and assume that it does not merely reduce to the complex scalars, that is to say that there do exist regular elements in $P$ of positive degree.

It turns out that the integer $t_{0}$ may be taken to be independent of the choice of the regular element $p_{2}$ and that we may associate with $p_{1} / p_{2}$ a linear space endomorphism of each graded component $N^{(t)}$ for $t \geqq t_{0}$. This endomorphism, taking $t=t_{0}$, is specified by

$$
N^{\left(t_{0}\right)} \stackrel{p_{2}^{-1}}{\longrightarrow} N^{\left(t_{0}+m\right)} \stackrel{p_{1}}{\longrightarrow} N^{\left(t_{0}\right)} .
$$


Here we have denoted the degree of $p_{2}$ (and $p_{1}$ ) by $m$. If the resulting collection of endomorphisms of $N^{\left(t_{0}\right)}$ be denoted by $R_{1}$, we can say that we have a homomorphism of the algebra $R$ of formal admissible fractions onto a commutative algebra $R_{1}$ of linear space endomorphisms of $N^{\left(t_{0}\right)}$. In the standard case, as also in the case of linear parameters, one has $t_{0}=0$, and $R_{1}$ is a collection of endomorphisms of the given space $G$. In the case of the operator-bundle (4.2) we have, apart from singular cases, $t_{0}=n-1$. We get a functional calculus in which formal fractions are mapped into endomorphisms of $N^{(n-1)}$. As noted above, the latter is simply the collection of all polynomials in two homogeneous variables, of degree $n-1$, with coefficients in $G$. It is thus isomorphic to the direct sum of $n$ copies of $G$. Here we are beginning to make contact with the "multiple completeness," or " $n$-fold completeness" property of operator-bundles, according to which eigenfunction expansions are to be sought in a direct sum space [44], [73].

To get expansion theorems by the present method, one would have to express the algebra $R_{1}$ as a symmetric algebra [74], which in turn involves duality relations and products. Without going so far let us note that in the normed case in which $G$ is a complex Banach space, the algebra $R_{1}$ may be completed to a Banach algebra. We may assume, though this is a little too much, that all operator-coefficients in the $D_{r}$ are continuous, and norm the graded components of $Q(G)$ and $N$ by crudely adding the norms of the coefficients in the polynomials concerned. Then our regular fractions, if nontrivial regular fractions exist, will be continuous, and the algebra may be completed in the operator norm. We may then identify the maximal ideals with fractions which vanish at points of a certain collection of nonzero complex $(k+1)$-tuples. An element of $P$ will be regular if it does not vanish at these points.

7. Eigenvalues and ideals. As already mentioned in $\$ 2$ for a more special case, we may for (5.1) define an eigenvalue as a nonzero $(k+1)$-tuple $\left(\lambda_{0}, \cdots, \lambda_{k}\right)$ such that the $k$ linear space operators $D_{r}\left(\lambda_{0}, \cdots, \lambda_{k}\right)$ all have nonzero kernel, or again such that the $k$ induced operators $D_{r}^{\dagger}\left(\lambda_{0}, \cdots, \lambda_{k}\right)$ have kernels, subspaces of $G$, with nonzero intersection. The latter gives a natural idea of an eigensubspace. This is all very well but leaves us without any extension of the idea of a root-subspace or of that of the rank of an eigenvalue. It is appropriate to recall that in algebraic geometry the intersections of a collection of varieties may not be adequately described by a specification of the points of intersection; likewise, in polynomial algebra, an 
ideal need not be specified completely by a knowledge of the common zeros of its members.

To express the needed concepts in the language of partial differential equations, we must inquire into special relations which may exist between $N$, the collection of solutions of (5.4), and an ideal, $\omega$ say, in $P$. We confine attention to homogeneous ideals in the graded algebra $P$, the original expressions (5.1) also being homogeneous. The elements of $\omega$, as other elements of $P$, are currently being interpreted as partial differential operators acting formally on polynomials. One obvious question is whether $N$ contains nonzero elements of all degrees, which are annihilated by all elements of $\omega$ acting as differential operators. Again, we may ask whether $\omega N$, the subset of $N$ generated by products of elements of $\omega$ and $N$, is actually a strict subset of $N$ or not. These questions have an obvious and genuine analogy with those in the standard case in which we ask whether $A-\lambda I$, for given scalar $\lambda$, has nonzero kernel or cokernel. More questions of the same kind can be asked. Generally, we are interested in the triviality or otherwise of $\operatorname{Ext}_{P}^{r}(P / \omega, N), r=0,1, \cdots$, and the relations between them.

In the finite-dimensional case (2.7) it is appropriate to define ann $N$, the ideal in $P$ of elements which annihilate all elements of $N$. According to a modified Cayley-Hamilton theorem, ann $N$ includes the ideal generated by the polynomials $\operatorname{det} D_{r}\left(\xi_{0}, \cdots, \xi_{k}\right)$; here the determinant is evaluated by choosing bases in $G_{r}, H_{r}$. As in the standard matrix case, this ideal may be used to obtain decompositions. A similar ideal is used in connection with more general systems of partial differential equations [77].

Let us now introduce a concept similar to that of the rank of an eigenvalue. For a given ideal, we may consider the subsets of $N$ annihilated by $\omega$, by $\omega^{2}, \cdots$, and so on. These form, of course, a sequence of increasing subsets; a convenient notation is

$$
\operatorname{Hom}_{P}(P / \omega, N) \subset \operatorname{Hom}_{P}\left(P / \omega^{2}, N\right) \subset \cdots .
$$

Here, in our homogeneous formulation, we consider that the inclusion is strict if it is strict in graded components of arbitrarily high degree. The inclusions are either all strict or strict up to some point only. The least $r$, if any, such that $\operatorname{Hom}_{P}\left(P / \omega^{r}, N\right)$ and $\operatorname{Hom}_{P}$ $\left(P / \omega^{r+1}, N\right)$ coincide above some graded component may be taken to be the rank.

If $k>1$, we have a variety of kinds of ideal deserving consideration, notably maximal, prime, primary and principal. In the algebraically closed case, a maximal ideal in $P$ is the collection of polynomials vanishing at some scalar $(k+1)$-tuple; as always, we confine our- 
selves to homogeneous ideals. If $\omega$ is a maximal ideal and we ask whether $\omega$ annihilates nonzero elements of $N$ in all degrees, we are repeating our previous definition of an eigenvalue.

In the case of a principal ideal, generated by $p \in P$, the questions to be asked are whether $p$ acting on $N$ has nontrivial kernel and again whether it is an epimorphism. These questions arose in the definition in $\$ 6$ of a "regular" element. If $p$ is not regular, that is to say if $p: N \rightarrow N$ has nontrivial kernel or $p N$ is a strict subset of $N$, we proceed to the same questions for the iterates of $p$, or $p^{r}$. If the answers are ultimately constant for large $r$, we proceed to a direct sum decomposition.

The programme of amassing information of all kinds in the relation of an ideal in $P$ acting differentially on $N$ provides an example of pointwise spectral theory. As in the special case of the study of indices, root-spaces and so on, it may be commended as worthy of interest in itself, even without any attempt to define a spectrum and to attach global or structural properties to it [43].

There is perhaps no need to attempt any definition of the spectrum which should remain good for all time. Let us rather say, to begin with, that we are concerned with topological spaces of homogeneous ideals in $P$ and with the properties of sheaves which may be defined over them. The collection of such topological spaces forms, of course, a category with continuous injections as morphisms. We are always at liberty to use the discrete topology, and others, not necessarily Hausdorff, may be defined without reference to a metric, such as the hull-kernel or Zariski topologies [31], [95]. In any given setting, we ask for the strongest or weakest topology to produce a given result, as appropriate. The comparison of various topologies on the maximal ideals is well known in normed ring theory [74], [80]. We stand here at a point of confluence of algebraic tendencies in analysis and developments in algebra proper and algebraic geometry.

To indicate how sheaves come in, suppose we have a collection $\Omega$ of ideals in $P$, with, for simplicity, the discrete topology. For any $u \in N$, we define its support as the collection of $\omega \in \Omega$ such that $u \notin \omega N$; for the topic of modules with supports, I refer to [19]. The natural question is whether an element with empty support must be zero or whether, in the terminology of [19], the complex formed by this module with supports is "separated"; more in the direction of spectral resolution, one asks whether it is "fine." The next step is to ask whether the images of $u \in N$ in $N / \omega N$ can be prescribed at will, or rather for what collections $\Omega$ this is possible. To be more formal about this we should set up a presheaf [52] or stack. Whatever the 
topology on $\Omega$, with every open $U \subset \Omega$ we associate a module; each $u \in N$ determines an element of this module, being the map from $\omega$ to the image of $u$ in $N / \omega N$. In pointwise and local spectral theory we are concerned with the stalks of the resulting sheaf, in global spectral theory with the global properties of this sheaf, and in its relation to the stack.

In the above we should add to the great variety of possible questions arising from the choice of ideals and topologies, the further dimension arising from the possible choice of other modules associated with (5.1). We have, for example, not touched on the apparatus of fractions leading to holomorphic functions.

8. Duality. So far I have concentrated on the module $N$ of solutions of the partial differential equations (5.1), since this accommodates much that is usual in the standard case. However, there are a great many others, arising by way of duality or resolutions, or by application of standard functors. Here I mention one that arises by way of linear space duality. It is to be assumed that with each linear space we can associate a dual or conjugate, indicated by $\left({ }^{*}\right)$, as for example in the finite-dimensional, Hilbert space or algebraic cases. The operator coefficients in $D_{r}$, given as acting from $G_{r}$ to $H_{r}$, will have induced actions from $H_{r}^{*}$ to $G_{r}^{*}$ and further induced actions on

$$
H^{*}=H_{1}^{*} \otimes \cdots \otimes H_{k}^{*} \text {. }
$$

We can therefore define a second module $N^{*}$ as the set of solutions of

$$
D_{r}\left(\partial / \partial x_{0}, \cdots, \partial / \partial x_{k}\right) u^{*}=0, \quad u^{*} \in Q\left(N^{*}\right) .
$$

Naturally, the considerations of $\S \S 5-7$ may be applied also to $N^{*}$, though not necessarily producing any essential novelty.

The utility of $N^{*}$ lies also in certain orthogonality relations. In the absence of assumptions of a hermitian character, we should strictly speaking talk of biorthogonality. Special elements of $N$ and of $N^{*}$ will, in a certain sense, be orthogonal to each other. This becomes orthogonality in the usual sense when we have a conjugacy operation from $N$ to $N^{*}$.

I shall not go into the latter and merely want to indicate verbally how the orthogonality is expressed. We consider elements of $N$ as polynomials in variables $x_{s}$ with coefficients in $G$ and elements of $N^{*}$ as polynomials in a duplicate set of variables $x_{s}^{\prime}$ with coefficients in $H^{*}$. We set up a product of elements in $N^{*}$ and $N$ whose value is a polynomial in all these variables with scalar coefficients. We are now 
dealing with $P \otimes P$-modules in which elements of $P \otimes P$ act as differential operators in all $2 k+2$ variables.

In fact, a product of this kind is given by a differential operator in all the variables, with coefficients in $\operatorname{Hom}(G, H)$. Not all such products are relevant, however. We want only those associated with differential operators which yield orthogonality relations. For this the following is sufficient. If, replacing $\partial / \partial x_{s}, \partial / \partial x_{s}^{\prime}$ by $\xi_{s}, \xi_{s}^{\prime}$, the operator in question is written

$$
E\left(\xi_{0}, \cdots, \xi_{k} ; \xi_{0}^{\prime}, \cdots, \xi_{k}^{\prime}\right),
$$

we require that the expressions

$$
E\left(\xi_{0}, \cdots, \xi_{k} ; \xi_{0}^{\prime}, \cdots, \xi_{k}^{\prime}\right)\left(\xi_{u} \xi_{\beta}^{\prime}-\xi_{\alpha}^{\prime} \xi_{\beta}\right)
$$

be expressible as linear combinations, with suitable coefficients of the $D_{r}^{\dagger}\left(\xi_{0}, \cdots, \xi_{k}\right), D_{r}^{\dagger}\left(\xi_{0}^{\prime}, \cdots, \xi_{k}^{\prime}\right)$. We have, indeed, a $P \otimes P$-module of suitable operators, and no uniquely preferred product.

9. Polynomial aspects. I shall now go back to the study of the operators (5.1) as expressions in terms of indeterminates. One must regard this aspect as basic and the most comprehensive. The partial differentiation interpretation we have been discussing is indeed approximately equivalent to the more algebraic interpretation to be discussed now; the two are related by duality, as applied to the polynomial algebra. However, such operations as replacing the indeterminates by complex scalars, forming tensor products with rings of fractions, are more directly connected with the interpretation in terms of indeterminates.

Before elaborating on the duality between polynomials and partial differential operators, a few words on the topic of polynomial algebras may be in place. We are here concerned with a monoid-algebra [62]. The monoid in question is formed by monomials $\prod_{0}^{k} \xi_{s}^{n_{s}}$ with nonnegative integral $n_{s}$. The monoid-algebra consists of formal linear combinations of these with coefficients in a field $K$; if we take the coefficients in a linear space over $K$, then we get a module over this polynomial algebra. A similar construction can be carried out in other cases also. Thus, a finite group gives rise to a group algebra; the representation theory of finite groups is concerned with modules over this algebra. I mention this in order to place this representation theory in alignment with what we are doing here [76].

Let $P$ denote the polynomial algebra in $\xi_{0}, \cdots, \xi_{k}$, and $P^{(n)}$ the subset formed by homogeneous polynomials of degree $n$. We want to place in its formal setting the device of considering elements of $P$ as 
partial differential operators acting on polynomials or formal power series in dummy variables $x_{0}, \cdots, x_{k}$. For this purpose we note that $\operatorname{Hom}_{K}(P, K)$ can be considered as a $P$-module, with elements of $P$ acting multiplicatively on the first argument. More specifically, $\operatorname{Hom}_{K}(P, K)$ can be considered as a direct sum of the finite-dimensional linear spaces $\operatorname{Hom}_{K}\left(P^{(n)}, K\right), n=0,1, \ldots$. An element $p \in P^{(m)}$ has a natural action $p: P^{(n)} \rightarrow P^{(n+m)}$ and an induced action $p: \operatorname{Hom}_{K}\left(P^{(n+m)}, K\right) \rightarrow \operatorname{Hom}_{K}\left(P^{(n)}, K\right)$. All this may be expressed otherwise by identifying $\operatorname{Hom}_{K}\left(P^{(n)}, K\right)$ with the set $Q^{(n)}$ of homogeneous polynomials in the $x_{8}$ with scalar coefficients, with the convention that an element of $P^{(n)}$ acts on an element of $Q^{(n)}$ as the corresponding differential operator. Thus $Q$, the graded $P$-module of polynomials in the $x_{s}$, appears as the dual to $P$; it is not, for us, an algebra and does not arise in any analytic manner.

I next take up the formal operations associated with (5.1). Let $P(S)$ denote the set of polynomials in the indeterminates $\xi_{s}$ with coefficients in any linear space $S$ over the field in question. It has an obvious grading into homogeneous components and is a $P$-module. We consider the objects (5.1) as defining homomorphisms of $P$ modules, namely

$$
D_{r}\left(\xi_{0}, \cdots, \xi_{k}\right): P\left(G_{r}\right) \rightarrow P\left(H_{r}\right), \quad r=1, \cdots, k .
$$

In this map we multiply polynomials in the usual way; the operator coefficients in $D_{r}$ will map vector coefficients in $P\left(G_{r}\right)$ into vector coefficients in $P\left(H_{r}\right)$. For formal purposes it is convenient to write this map as a formal complex of $P$-homomorphisms o $P$-modules

$$
0 \rightarrow P\left(G_{r}\right) \stackrel{D_{r}}{\rightarrow} P\left(H_{r}\right) \rightarrow 0, \quad r=1, \cdots, k .
$$

Similarly, if the coefficients in $D_{r}$ have their dual actions, we have

$$
0 \rightarrow P\left(H_{r}^{*}\right) \stackrel{D_{r}}{\rightarrow} P\left(G_{r}^{*}\right) \rightarrow 0, \quad r=1, \cdots, k .
$$

We must now form the tensor product of these complexes [66] of graded $P$-modules; this will of course depend on the precise choice of tensor product in the underlying linear spaces. In simple cases (9.29.3) are all monomorphic, and the homology of the resulting complex is accordingly rather simple, except at the last point, which is our main concern.

Space and time preclude my doing much more than indicating what this homology-module is. In the case of $(9.3)$ we denote by $M^{*}$ the quotient-module

$$
P\left(G^{*}\right) /\left[D_{1}^{\dagger}, \cdots, D_{k}^{\dagger}\right]
$$


Here we have taken the liberty of writing $G^{*}$ for $G_{1}^{*} \otimes \cdots \otimes G_{k}^{*}$. The denominator in (9.4) is to mean the submodule generated in $P\left(G^{*}\right)$ by $D_{1}^{\dagger}, \cdots, D_{k}^{\dagger}$, with $D_{r}^{\dagger}$ acting on $P\left(G_{1}^{*} \otimes \cdots \otimes H_{r}^{*} \otimes \cdots \otimes G_{k}^{*}\right)$. A module $M$ may be defined similarly in connection in (9.2). Here we follow the pattern of algebraic geometry [31], passing from a set of polynomials to the ideal which they generate and then to the quotient-ring modulo this ideal.

The full tensor products of (9.2-9.3), certain complexes of $P$ modules, are of interest in connection with direct sum or other decompositions of $M$ and $M^{*}$. In favourable cases, we obtain direct sum decompositions of these complexes.

I pass now to the connection with partial differential equations. We can interpret $N$ as an element of $\operatorname{Hom}_{P}\left(M^{*}, Q\right)$, and $N^{*}$ as an element of $\operatorname{Hom}_{P}(M, Q)$. In the former case, if $u \in N$, then for variable $\phi \in M^{*}$ and fixed $u$ we have the map from $\phi \in M^{*}$ to $\phi u \in Q$; here in forming $\phi u$ we interpret the indeterminates $\xi_{s}$ in $\phi$ as partial differential operators $\partial / \partial x_{s}$, to act on the variables $x_{s}$ in $u$. In addition, the vector coefficients in $M^{*}$, elements of $G^{*}$, act on the vector coefficients in $N$, elements of $G$, to give scalars. It is a matter of indifference which representative in $P\left(G^{*}\right)$ we choose for an element of $M^{*}$ since the difference between two such representatives annihilates $N$.

In the genuine theory of linear partial differential equations with constant coefficients, the module $Q$ of formal polynomials or power series is replaced by a more interesting collection of infinitely differentiable functions, and of course the differentiation is no longer formal. The technique of forming a quotient-module from a given set of operators, and then using its free resolution, has been used by Palamodov [77] in his work on " $M$-convexity."

10. Singular matrix pencils. I would like to make the above constructions more explicit in a rather simple case, that of a pencil

$$
D=A \xi+B \eta,
$$

where $A, B$ are homomorphisms from a linear space $G$ to a linear space $H$, both of finite dimension, not necessarily the same in each case. I now use $P$ to denote the algebra of polynomials in the indeterminates $\xi$, $\eta$, while $P(G)$, etc., will be the corresponding object with coefficients in $G$. In place of (9.2-9.3) we have the formal complexes

$$
\begin{aligned}
0 & \rightarrow P(G) \stackrel{D}{\rightarrow} P(H) \rightarrow 0, \\
0 & \rightarrow P\left(H^{*}\right) \stackrel{D}{\rightarrow} P\left(G^{*}\right) \rightarrow 0 .
\end{aligned}
$$


We have no immediate room for tensor products (though it is useful to tensor $(10.2-10.3))$. The homology-modules $(10.2-10.3)$ at the second points, or cokernels of $D$, are

$$
\begin{aligned}
M & =P(H) / D P(G), \\
M^{*} & =P\left(G^{*}\right) / P\left(H^{*}\right) D .
\end{aligned}
$$

Let further $Q$ denote the set of formal power series or polynomials in "variables" $x, y$, with a similar notation in the case of vectorial coefficients. Then $Q, Q(G)$, etc., are $P$-modules if $\xi, \eta$ act as $\partial / \partial x$, $\partial / \partial y$. Then $N, N^{*}$ are respectively the sets of solutions of the differential equations

$$
\begin{aligned}
D u & =0, & & u \in Q(G), \\
D u^{*} & =0, & & u^{*} \in Q\left(H^{*}\right) .
\end{aligned}
$$

In spectral theory, these commonly appear under another name. If we consider a typical element of $N$ in the form

$$
u=\sum_{0}^{n} g_{r} x^{n-r} y^{r}\left(\begin{array}{l}
n \\
r
\end{array}\right), \quad g_{r} \in G,
$$

then (10.6) is equivalent to

$$
A g_{0}+B g_{1}=0, \cdots, A g_{n-1}+B g_{n}=0 .
$$

Such objects are known as "chains," for the detailed theory of which I refer to the works cited in $\$ 2$. Much as in $\$ \S 6-7$, chains provide an extension to pencils of the construction of polynomials in an endomorphism.

Chains of a slightly different kind play a role in the reduction of singular matrix pencils, in which $D$ in $(10.2-10.3)$ need not be monomorphic $[42$, p. 35]. I should like to illustrate the present formalism by showing how this topic fits into it.

If we consider the kernel, $L^{*}$ say, of (10.3), we see that it is generated by homogeneous polynomials in $\xi, \eta$ with coefficients in $H^{*}$. In a typical case,

$$
\sum_{0}^{n} h_{r}^{*} \xi^{n-r} \eta^{r}(A \xi+B \eta)=0, \quad h_{r}^{*} \in H^{*},
$$

which is equivalent to

$$
h_{0}^{*} A=0, \quad h_{0}^{*} B+h_{1}^{*} A=0, \cdots, h_{n}^{*} B=0,
$$

which is the other kind of "chain" mentioned. When these chains are present at all, they have certain orthogonality relations with chains of the previously mentioned type. 
To clarify this, it is useful to consider $\operatorname{Tor}_{2}^{P}\left(M^{*}, K\right)$, where $K$ is the field, and is a $P$-module under the usual prescription that $\xi K$ $=\eta K=0$. We may calculate it either by means of the resolution

$$
0 \rightarrow L^{*} \rightarrow P\left(G^{*}\right) \rightarrow P\left(H^{*}\right) \rightarrow 0,
$$

as a left complex over $M^{*}$, or again, in the case of $K$, by means of

$$
0 \rightarrow P \rightarrow P \oplus P \rightarrow P \rightarrow 0 .
$$

Here the first nontrivial map takes $p$ into $(\xi p, \eta p)$ and the second takes $\left(p_{1}, p_{2}\right)$ into $\left(\eta p_{1}-\xi p_{2}\right)$. The first gives the elements of $L^{*}$ reduced modulo those generated by elements of $L^{*}$ of lower degree, while the second gives elements of $M^{*}$ which are annihilated by multiplication by $\xi$ and also by $\eta$. A more detailed argument shows that the connecting homomorphism reduces degrees by 1 ; we have an isomorphism between elements $L^{*}$ of degree $n+1$ taken modulo those generated by elements of $L^{*}$ of lower degree and by elements of $M^{*}$, annihilated by $\xi$ and by $\eta$, of degree $n$. The latter in turn yield a special kind of element of $N$, namely those which cannot be generated by differentiation of higher order elements of $N$. We derive a product between special elements of $L^{*}$ and special elements of $N$, in which we go from $L^{*}$ to $M^{*}$ by means of the connecting homomorphism, and then apply the result to elements of $N$, more specifically to elements of $\operatorname{Ext}_{P}^{2}(K, N)$.

As usual, the topic of products has further ramifications. For $v^{*} \in L^{*}, u \in N$, we may interpret $v^{*} A u, v^{*} B u$ as polynomials in $\xi, \eta$, $x, y$ with scalar coefficients without, that is, making $\xi, \eta$ act differentially. We have therefore maps

$$
L^{*} A N, L^{*} B N \rightarrow P \otimes_{K} Q,
$$

which are homomorphisms of $P \otimes_{K} P$-modules (the second factor acts differentially); the differential machinery is creaking slightly here. It then turns out that the images in these maps have a highly special form, namely annihilated by skew-symmetric elements of $P \otimes_{K} P$ or by $(\eta \partial / \partial x-\xi \partial / \partial y)$ (see e.g. [42, pp. 35-42]).

11. Determinantal procedures. I should like to move away from problems in the polynomial area and consider briefly some points which arise in connection with the array (2.1). It is natural, in view of the special case in which the $G_{r}$ are all one-dimensional, to apply techniques involving determinants, minors, and cofactors. Here we mean determinants with operator entries.

Let us pose the extension of the problem of the solution of $k$ scalar inhomogeneous equations in $k$ unknowns. We start with an array 
$A_{r s}$, as in (2.1), but with $1 \leqq r, s \leqq k$. Again, we assume that $A_{r s}: G_{r} \rightarrow H_{r}$ is a linear operator and denote by $A_{r s}^{\dagger}$ the induced operator acting from (2.4) to the $r$ th of the spaces (2.5). In the problem in question we suppose given $h_{r} \in G_{1} \otimes \cdots \otimes H_{r} \otimes \cdots \otimes G_{k}, r=1, \cdots, k$, and ask for $g_{1}, \cdots, g_{k} \in G$ such that

$$
\sum_{s=1}^{k} A_{r s}^{\dagger} g_{s}=h_{r}, \quad r=1, \cdots, k .
$$

We now follow the procedure of Cramer's rule. Let $\Delta$ denote the determinant of the $A_{r s}^{\dagger}$, the operators being multiplied according to operator-composition, or for that matter the determinant of the $A_{r s}$ with tensorial multiplication. The value of $\Delta$ will be a homomorphism from $G$ to $H$. We denote by $\Delta_{r s}$ the cofactor of $A_{r s}$ which will map from $G_{1} \otimes \cdots \otimes H_{r} \otimes \cdots \otimes G_{k}$ into $H$. On applying, for any fixed $s$, the operators $\Delta_{r s}$ to (11.1) and summing over $r$, we obtain

$$
\Delta g_{s}=\sum_{s=1}^{k} \Delta_{r s} h_{r}, \quad s=1, \cdots, k .
$$

It follows from this that if $\Delta$ is an isomorphism, then (11.1) has at most one solution, given by (11.2).

However, in contradistinction to the scalar case, we cannot check this solution by substitution and rearrangement of factors. The necessary identity is

$$
\begin{aligned}
\sum A_{r s}^{\dagger} \Delta^{-1} \Delta_{u s} & =0 & & (r \neq u) \\
& =I_{r} & & (r=u)
\end{aligned}
$$

where $I_{r}$ denotes the identity on $G_{1} \otimes \cdots \otimes H_{r} \otimes \cdots \otimes G_{k}$. Here it is not permissible to rearrange the factors on the left of (11.3).

The conclusion that if $\Delta$ is an isomorphism, then (11.3) hold, and so Cramer's rule in this setting, is easily established by dimensional arguments if (2.7) holds.

A similar problem arises for the full array (2.1) when we ask for the general solution of

$$
\sum_{s=0}^{k} A_{r s}^{\dagger} g_{s}=0, \quad r=1, \cdots, k .
$$

In this case, let $\Delta_{s}$ denote the determinant, a homomorphism from $G$ to $H$, obtained from this array by omitting the column containing $A_{1 s}$ multiplied by $(-1)^{8}$. Let us assume that at least $\Delta_{0}$ is an isomorphism (any linear combination of the $\Delta_{s}$ would do as well). We 
then have as a consequence of (11.4) that

$$
g_{s}=\Delta_{0}^{-1} \Delta_{s} g_{0}
$$

which specifies $g_{1}, \cdots, g_{k}$ in terms of $g_{0}$. The question is now whether (11.5) gives a solution with $g_{0}$ arbitrary, and again dimensional considerations suffice in the finite-dimensional case. We are here concerned with whether the operators $\Delta_{0}^{-1} \Delta_{s}, s=1, \cdots, k$, all commute as endomorphisms of $G$ [9]. One may express (11.4-11.5) in terms of partial differential operators and indeed as a microscopic part of a rather large diagram.

There are many other problems regarding decomposability which admit a ready solution in the finite-dimensional case. Thus, for (11.1), one asks whether, if $\Delta$ is singular, i.e. has nonzero kernel, its kernel contains nonzero decomposable tensors, and this is true if (2.7) holds [10]. A similar situation holds regarding positive-definiteness on decomposable tensors for hermitian operators on finite-dimensional Hilbert spaces.

\section{REFERENCES}

1. Dž. E. Alłahverdiev, Completeness of the system of eigenelements and adjoined elements of a class of nonselfadjoint operators depending on a parameter, Dokl. Akad. Nauk SSSR 160 (1965), 1231-1234 = Soviet Math. Dokl. 6 (1965), 271-275.

2. P. M. Anselone, Matrices of linear operators, Enseignement Math. (2) 9 (1963), 191-197.

3. N. Aronszajn and U. Fixman, Algebraic spectral problems, Technical Report No. 11, New Series, Lawrence, Kansas, 1967.

4. - Proc. Intern. Sympos. on Linear Spaces (Jerusalem, 1960), Jerusalem Academic Press, Jerusalem and Pergamon Press, Oxford, 1961.

5. F. M. Arscott, Two-parameter eigenvalue problems in differential equations, Proc. London Math. Soc. (3) 14(1964), 459-470.

6. - A treatment of the ellipsoidal wave equation, Proc. London Math. Soc. (3) 9 (1959), 21-50.

7. - Periodic differential equations, Macmillan, New York, 1964.

8. F. V. Atkinson, Boundary problems leading to orthogonal polynomials in several variables, Bull. Amer. Math. Soc. 69 (1963), 345-351.

9. - Multivariate spectral theory: the linked eigenvalue problem for matrices, Technical Summary Report, No. 431, U. S. Army, Math. Research Center, Madison, Wis., 1964.

10. - Singularity of determinantal endomorphisms, Abstract 619-85, Notices Amer. Math. Soc. 12 (1965), 81.

11. Discrete and continuous boundary problems, Academic Press, New York, 1964.

12. M. Audin, Sur les équations linéaires dans un espace vectoriel, Ph.D. Thesis, Paris, 1957.

13. R. Bellman, Introduction to matrix analysis, McGraw-Hill, New York, 1960. 
14. Ju. M. Berezanskii, Expansions in eigenfunctions of selfadjoint operators, Mat. Sb. 43(85) (1957), 75-126. (Russian)

15. - Expansion in eigenfunctions of selfadjoint operators, Naukova Dumka, Kiev, 1965. (Russian)

16-18. M. Bôcher, The theorems of oscillation of Sturm and Klein. I, Bull. Amer. Math. Soc. 4 (1897-1898), 295-313; II, ibid., 365-376; III, ibid. 5 (1898-1899), 22-43.

19. A. Borel, Cohomologie des espaces localement compacts d'après J. Leray, Lecture Notes in Math., No. 2. Springer-Verlag, Berlin, 1964.

20. A. Brown and C. Pearcy, Spectra of tensor products of operators, Proc. Amer. Math. Soc. 17 (1966), 162-166.

21. C. C. Camp, An expansion involving p inseparable parameters associated with a partial differential equation, Amer. J. Math. 50 (1928), 259-268.

22. - On multiparameter expansions associated with a differential system and auxiliary conditions at several points in each variable, Amer. J. Math. 60 (1930), 447452.

23. R. D. Carmichael, Boundary value and expansion problems: Algebraic basis of the theory, Amer. J. Math. 43 (1921), 69-101.

24. - Boundary value and expansion problems: Formulation of various transcendental problems, Amer. J. Math. 43 (1921), 232-270.

25. - Boundary value and expansion problems: Oscillatory, comparison and expansion problems, Amer. J. Math. 44 (1922), 129-152.

26. R. Carroll, Problems in linked operators. I, Math. Ann. 151 (1963), 272-282.

27. H. Cartan and S. Eilenberg, Homological algebra, Princeton Univ. Press, Princeton, N. J., 1956.

28. L. A. Coburn and A. Lebow, Algebraic theory of Fredholm operators, J. Math. Mech. 15 (1966), 577-584.

29-30. H. O. Cordes, Über die Spektralzerlegung von hypermaximalen Operatoren, die durch Separation der Variablen zerfallen. I, Math. Ann. 128 (1954), 257-289; II, Math. Ann. (1955), 373-411.

31. J. Dieudonné, Algebraic geometry, Dept. of Math. Lecture Notes, No. 1, University of Maryland, College Park, Md., 1962.

32. H. P. Doole, $A$ certain multi-parameter expansion, Bull. Amer. Math. Soc. 37 (1931), 439-446.

33. N. Dunford, Spectral operators, Pacific J. Math. 4 (1954), 321-354.

34. - A spectral theory for certain operators on a direct sum of Hilbert spaces, Math. Ann. 162 (1965/1966), 294-330.

35. M. G. Džavadov, On the completeness of certain eigenfunctions of a nonselfadjoint differential operator, Dokl. Akad. Nauk SSSR 159 (1964), 723-725 = Soviet Math. Dokl. 5 (1964), 1571-1573.

36. A. Erdelyi, et al., Higher transcendental functions. Vol. II, McGraw-Hill, New York, 1953.

37. M. Faierman, Boundary value problems in differential equations, Thesis, Toronto, 1966.

38. E. Fischer, Über die Differentiationsprozesse der Algebra, J. Reine Angew. Math. 148 (1917), 1-78.

39. U. Fixman, On algebraic equivalence between pairs of linear transformations, Trans. Amer. Math. Soc. 113 (1964), 424-453.

40. M. M. Flood, Equivalence between pairs of matrices, Trans. Amer. Math. Soc. 44 (1938), 8-17.

41. B. Friedman, $A n$ abstract formulation of the method of separation of variables, 
Proc. Conference on Differential Equations, Univ. of Maryland Book Store, College Park, Md., 1956, pp. 209-226.

42. F. R. Gantmaher, Applications of the theory of matrices, English version, Interscience, New York, 1959.

43. I. C. Gohberg and M. G. Krein, Basic propositions on defect-numbers, rootnumbers and indices of linear operators, Uspehi Mat. Nauk 12 (1957), no. 2(74), 43118; English transl., Amer. Math. Soc. Transl. (2) 13 (1960), 185-264.

44. - Introduction to the theory of linear nonselfadjoint operators in Hilbert space, "Nauka," Moscow, 1965. (Russian)

45. S. Goldberg, Unbounded linear operators: theory and applications, McGrawHill, New York, 1966.

46. W. Greub, Linear algebra, 3rd ed., Springer-Verlag, New York, 1967.

47. W. Gröbner, Moderne algebraische Geometrie, Springer-Verlag, Wien, 1949.

48. A. Grothendieck, Produits tensoriels topologiques et espaces nucleaires, Mem. Amer. Math. Soc. No. 16 (1955).

49. M. Hamermesh, Group theory and its application to physical problems, AddisonWesley, Reading, Mass., 1962.

50. E. Hilb, Über Kleinsche Theoreme in der Theorie der linearen Differentialgleichungen. II, Math. Ann. 68 (1910), 24-74.

51. D. Hilbert, Grundzïge einer allgemeinen Theorie der linearen Integralgleichungen, Berlin, 1912.

52. F. Hirzebruch, Topological methods in algebraic geometry, Springer-Verlag, New York, 1963.

53. L. Hörmander, Linear partial differential operators, Academic Press and Springer-Verlag, New York, 1963.

54. J. Horváth, Basic sets of polynomial solutions for partial differential equations, Proc. Amer. Math. Soc. 68 (1962), 204-209.

55. E. L. Ince, Ordinary differential equations, reprint, Dover, New York, 1944.

56. S. Karlin and J. MacGregor, Determinants of orthogonal polynomials, Bull. Amer. Math. Soc. 68 (1962), 204-209.

57. T. Kato, Perturbation theory for nullity, deficiency and other quantities of linear operators, J. Analyse Math. 6 (1958), 261-322.

58. M. V. Keldyš, On the eigenvalues and eigenfunctions of certain classes of nonselfadjoint equations, Dokl. Akad. Nauk SSSR 77 (1951), 11-14. (Russian)

59. F. Klein, Gesammelte Math. Abh. II, Berlin, 1922.

60. M. G. Krein and H. Langer, Certain mathematical principles of the linear theory of damped oscillations of continua, Appl. Theory of Functions in Continuum Mechanics (Proc. Internat. Sympos., Tbilisi, 1963), "Nauka," Moscow, 1965, pp. 283322. (Russian)

61. L. Kronecker, Algebraische Reduktion der Schaaren bilinearer Formen, S.-B. Akad. Berlin, 1890, 763-766.

62. S. Lang, Algebra, Addison-Wesley, Reading, Mass., 1965.

63. H. Langer, On invariant subspaces of linear operators acting in a space with indefinite metric, Dokl. Akad. Nauk SSSR 169 (1966), 12-15 = Soviet Math. Dokl. 7 (1966), 849-852.

64. R. E. Langer, Developments associated with a boundary problem not linear in the parameter, Trans. Amer. Math. Soc. 25 (1923), 155-172.

65. - The boundary problem associated with a differential system rational in the parameter, Trans. Amer. Math. Soc. 32 (1930), 238-259.

66. S. Mac Lane, Homology, Springer-Verlag, New York, 1963. 
67. A. I. Mal'cev, Foundations of linear algebra, OGIZ, Moscow, 1948; 2nd ed., 1956; English transl., Freeman, San Francisco, Calif., 1963.

68. A. S. Markus, Multiple completeness and convergence or multiple expansions in systems of eigenvectors and adjoined vectors of an operator-bundle, Dokl. Akad. Nauk SSSR 163 (1965), 1061-1064 = Soviet Math. Dokl. 6 (1965), 1062-1066.

69. J. Meixner and F. W. Schäfke, Mathieusche Funktionen und Sphäroidfunktionen, Springer-Verlag, Berlin, 1954.

70. P. H. Müller, Eine neue Methode sur Behandlung nichtlinearer Eigenwertaufgaben, Math. Z. 70 (1959), 381-406.

71. - Eigenwertabschätzungen für Gleichungen vom $T y p\left(\lambda^{2} I-\lambda A-B\right) x=0$, Arch. Math. 12 (1961), 307-310.

72. F. J. Murray and J. von Neumann, On rings of operators, Ann. of Math. (2) 37 (1936), 116-229.

73. M. A. Naimark, Spectral analysis of nonselfadjoint operators, Uspehi Mat. Nauk 11 (1956), no. 6(72), 183-208; English transl., Amer. Math. Soc. Transl. (2) 20 (1962), 55-75.

74. - INormed rings, English version, Noordhoff, Groningen, 1964.

75. D. J. Newman and H. S. Shapiro, Certain Hilbert spaces of entire functions, Bull. Amer. Math. Soc. 72 (1966), 971-977.

76. D. G. Northcott, An introduction to homological algebra, Cambridge Univ. Press, New York, 1960.

77. V. P. Palamodov, On the problem of $M$-convexity, Dokl. Akad. Nauk SSSR 161 (1965), 1015-1018 = Soviet Math. Dokl. 6 (1965), 550-554.

78. Anna J. Pell, Linear equations with two parameters, Trans. Amer. Math. Soc. 23 (1922), 198-211.

79. R. G. D. Richardson, Theorems of oscillation for two linear differential equations of the second order with two parameters, Trans. Amer. Math. Soc. 13 (1912), 22-34.

80. C. E. Rickart, General theory of Banach algebras, University Series in Higher Math., Van Nostrand, Princeton, N. J., 1960.

81. S. Rolewicz and D. Przeworska-Rolewicz, Equations in linear spaces, Monografie Matematyczne, PWN, Warsaw, 1967.

82. A. F. Ruston, Operators with a Fredholm theory, J. London Math. Soc. 29 (1954), 318-326.

83. P. Saphar, Contribution d l'étude des applications linéaires dans un espace de Banach, Bull. Soc. Math. France 92 (1964), 363-384.

84. F. W. Schäfke, Über Eigenwertaufgaben mit zwei Parametern, Math. Nachr. 6 (1951), 109-124.

85. R. Sikorski, The determinant theory in Banach space, Colloq. Math. 8 (1961), 141-198.

86. A. E. Taylor, Linear operators which depend analytically on a parameter, Ann. ff Math. (2) 39 (1938), 574, 593.

87. - Theorems on ascent, descent, nullity and defect of linear operators Math. Ann. 163 (1966), 18-49.

88. H. W. Turnbull and A. C. Aitken, An introduction to the theory of canonical matrices, Blackie, London, 1932. 1940.

89. B. L. van der Waerden, Moderne Algebra. vol. II, Springer-Verlag, Berlin,

90. E. B. van Vleck, On the polynomials of Stieltjes, Bull. Amer. Math. Soc. 4 (1897-1898), 426-438. 
91. E. T. Whittaker and G. N. Watson, Modern analysis, 4th ed., Cambridge Univ. Press, New York, 1958.

92. F. Wolf, Analytic perturbation of operators in Banach spaces, Math. Ann. 124 (1952), 317-333.

93. J. Yoshikawa, Dreiparametrige Randwertaufgaben, Göttingen Nachrichten, $1910,562-585$.

94. - Ein zweiparametriges Oscillationstheorem, Göttingen Nachrichten, $1910,586-594$.

95. O. Zariski and P. Samuel, Commutative algebra. I, II, Van Nostrand, Princeton, N. J., 1958, 1960.

UNIVERSITY OF TORONTO 